\title{
La lectura en segundas lenguas: análisis de la efectividad de la contextualización previa y sus implicaciones metodológicas
}

\author{
María Fernández Agüero \\ Universidad Autónoma de Madrid \\ Susana Montero Méndez \\ Centro Superior de Estudios Universitarios La Salle
}

Recibido: 29 marzo 2004 / Versión aceptada: 21 julio 2004

\begin{abstract}
RESUMEN: Este artículo presenta una investigación llevada a cabo con noventa estudiantes de inglés como segunda lengua con el fin de medir la incidencia de la contextualización a través del título como estrategia metodológica previa a la lectura de textos auténticos en segundas lenguas. En primer lugar se revisa la literatura existente y en particular la Teoría del Esquema, la cual pone de manifiesto la efectividad de dicha estrategia. Posteriormente, tras la descripción detallada de las características y el diseño del experimento, se exponen los resultados obtenidos; éstos corroboran la existencia de una mejora significativa en la comprensión del texto en presencia del título original, que resulta, por tanto, un elemento activador de los esquemas necesarios para la comprensión. La traducción del título al español, sin embargo, no supone un incremento significativo de la comprensión. Por último, se extraen conclusiones, se consideran las posibles implicaciones metodológicas y se sugieren posibles vías de continuación de la investigación. Palabras clave: habilidades lectoras, aprendizaje de segundas lenguas, inglés como segunda lengua, Teoría del Esquema, contextualización.
\end{abstract}

ABSTRACT: This article presents an experiment carried out with ninety students of English as a second language in order to measure the impact of contextualising through the title as a methodological strategy prior to reading in the comprehension of authentic texts in second languages. Firstly, we revise the existing literature and, in particular, the Schema Theory, which highlights the effectiveness of such strategy. Then, after a detailed description of the characteristics and design of the experiment, we present the results obtained; these corroborate the existence of a significant improvement in the comprehension of the text when the original title is present, thus proving that the title activates the squemata necessary for comprehension. Translating the title into Spanish does not lead to a significant increase in comprehension, though. Finally, we draw conclusions, consider the possible methodological implications and suggest possible further research.

Key words: reading skill, second language learning, English as a second language, Schema Theory, contextualisation. 


\section{INTRODUCCIÓN: LA LECTURA EN SEGUNDA LENGUAS}

En esta sección trataremos de los procesos presentes en la lectura de textos en una segunda lengua a través de la presentación de literatura relevante relacionada con el tema, haciendo especial referencia a la Teoría del Esquema ${ }^{1}$ y al papel que desempeña el título en la comprensión del texto en relación con dicha teoría.

La lectura de un texto debe ser tanto eficaz como eficiente. En palabras de Goodman y Goodman (1978: 133): «Readers and listeners are effective when they succeed in constructing meaning and are efficient when they use the minimal effort necessary». Sin embargo, tales eficacia y eficiencia no son fáciles de conseguir porque la destreza en la lectura implica la activación de procesos psicolingüísticos complejos a varios niveles: leer requiere reconocer e interpretar las palabras del mensaje escrito, tanto a nivel gramatical como semántico, y reaccionar ante el mensaje de alguna manera, por ejemplo elaborando conclusiones acerca de su validez. Incluso en una primera lengua, llegar a este punto de madurez en la lectura requiere un proceso largo y estructurado, que conlleva el aprendizaje y la práctica de todos los componentes del proceso de lectura por separado (Carroll, 1978).

Algunos expertos consideran que los modelos de lectura en una primera lengua difieren de los de la segunda (Eskey, 1988). Sin embargo, en un estudio con hablantes de chino, español e inglés, Block (1986) demuestra que las estrategias que usan los hablantes no nativos de inglés al leer un texto no difieren de las de los nativos. Sus resultados sugieren que el lector no nativo aplica al texto sus conocimientos del proceso de lectura en su propia lengua y así, existe una relación proporcional entre el uso eficaz de estrategias y la capacidad de aprendizaje. La conexión entre uso eficaz de estrategias y nivel de competencia en la segunda lengua no parece ser tan determinante, partiendo de un nivel mínimo de competencia necesario -en inglés, threshold level- (Devitt, 1997). Más recientemente, Fecteau (1999) también resalta la necesidad de tener en cuenta las habilidades lectoras del aprendiz en su propia lengua materna a la hora de analizar la lectura de literatura en una segunda lengua.

Las aportaciones de las dos últimas autoras tienen al menos dos implicaciones directas en la clase de inglés como segunda lengua: por un lado, el alumno será más o menos eficaz y eficiente en la lectura de textos ingleses en la medida en que lo sea en la lectura de textos en español. Por otro, puede llegar a la comprensión satisfactoria de un texto en inglés aún con un nivel bajo de competencia lingüística.

Sin embargo, es fácil imaginar una situación en la que el alumno sea un lector hábil en su lengua materna y aún así no llegue a la comprensión satisfactoria del texto en su segunda lengua. Parece razonable afirmar que este problema radica en que el texto, entonces, presenta uno o más rasgos, lingüísticos o no, que el alumno, por su condición de hablante no nativo, desconoce. Un enfoque que ayuda a entender el origen de dicho problema es la Teoría del Esquema.

${ }^{1}$ En inglés, Schema Theory. 


\subsection{La Teoría del Esquema en la lectura en segundas lenguas}

De acuerdo con la Teoría del Esquema, el significado de un texto no es inherente a éste y está fijado de manera inmutable, sino que depende del lector. Por un lado, entra en juego lo que el lector espera encontrar en el texto según sus conocimientos anteriores a la lectura del texto ${ }^{2}$. Por otro lado, nos encontramos con lo que el lector realmente extrae de la lectura del texto, siempre de acuerdo con esos conocimientos anteriores. La interacción de éstos con la nueva información del texto es lo que conduce a la comprensión (Anderson y Pearson, 1984). Cada lector individual, entonces, desempeña un papel fundamental y único en la comprensión de un texto dado.

Profundizando en el significado del conocimiento previo, la Teoría del Esquema sostiene que el proceso que se sigue al leer un texto es el siguiente: éste se interpreta contrastando cada información extraída de él con unas estructuras de conocimiento abstractas ya existentes en la mente del lector -porque son creadas a partir de su experiencia- llamadas esquemas ${ }^{3}$. Los esquemas operan en la selección, interpretación e incluso distorsión de la información (Schwarz y Flammer, 1981). Para que se produzca la comprensión del texto, debe existir compatibilidad entre la información y el esquema activado para ella (Carrell y Eisterhold, 1983). En otras palabras, de todos los esquemas disponibles en la mente del lector, éste debe escoger el adecuado. Como consecuencia, la imposibilidad por parte del lector de activar el esquema que se necesita para esa información conduce a diferentes grados de falta de comprensión del texto.

Por otro lado, la interacción entre el texto y el conocimiento previo es recíproca, ya que la nueva información contenida en el primero puede modificar los esquemas existentes en la mente del lector si aquélla no es consistente con éstos (Anderson y Pearson, 1984: 48). A través del input que recibe en el texto, el aprendiz de una segunda lengua puede, pues, modificar los esquemas que ha creado acerca de ésta, pues la lengua es también un tipo de esquema (Eskey, 1988: 96); si dichas modificaciones son adecuadas, mediante la lectura mejorará su competencia lingüística y se potenciarán las estrategias de aprendizaje que ayudan en esas modificaciones.

Los esquemas están organizados y unidos entre sí de maneras diversas, según se refieran a la estructura formal, retórica y organizativa de los textos o a su contenido. Algunos autores proponen clasificaciones de los esquemas sobre la base de esta distinción (Fillmore, 1981; Carrell, 1983). En el aula, la utilidad de estas taxonomías radica en que, antes de trabajar con un texto, el profesor se puede servir de ellas para predecir de una manera sistemática los esquemas que deben activarse en el camino hacia la comprensión; una vez identificados los esquemas, el profesor puede también potenciar su activación en la mente del alumno, e incluso crearlos, en una fase previa a la lectura. Esta fase no es, de ningún modo, obligatoria, por el mismo motivo por el que tampoco se puede asumir que los alumnos sólo deben leer acerca de lo que ya conocen (Silberstein, 1994). Junto con la información lingüística necesaria, puede que los alumnos sólo necesiten unos conocimientos previos mínimos para enfrentarse al texto, la explotación efectiva del cual depende, en última instancia, del buen criterio del profesor.

\footnotetext{
${ }^{2}$ En inglés, background knowledge.

${ }^{3}$ En inglés, schemata.
} 
En la enseñanza de segundas lenguas, resulta útil tener en cuenta la Teoría del Esquema a la hora de diseñar una metodología conducente al aprendizaje. Si no se consideran los conocimientos previos del alumno y no se contempla la posibilidad de que éstos difieran de los conocimientos del hablante nativo, no se comprenderá la naturaleza de los problemas de comprensión del alumno y, en definitiva, la metodología no será efectiva. Por otro lado, la Teoría del Esquema puede ser útil para la investigación en segundas lenguas o, más concretamente, en la detección y análisis de problemas que surgen en el aula de segundas lenguas. En este sentido, este estudio explora las dificultades que experimenta el lector no nativo ante la supresión de un contexto previo a la lectura, que permitiría la activación de esquemas antes de leer, y las consecuencias que esto tiene para la activación de los esquemas adecuados a la selección e interpretación de la información.

\subsection{La contextualización como elemento necesario en la lectura: el título}

El título de un texto no se debe ver en ningún caso como una simple etiqueta cuya única función es la de incitar a la lectura. En la clase de lenguas, junto con actividades de prelectura tales como las discusiones en grupo o la lluvia de ideas, los títulos sirven para que los alumnos se sitúen en el texto antes de enfrentarse a él y puedan predecir su contenido. A través del título, la contextualización previa a la lectura ayuda a la selección de los esquemas adecuados para la comprensión del texto. Según Schwarz y Flammer (1981: 61):

A [title] is expected to select and activate a schema from an already existing repertory of schemata and thus, to provide the reader with the general schemata at the beginning, to protect the elements from being dismissed before their contribution to the whole is understood, and to permit the reader to concentrate on the encoding of the important ideas.

La literatura corrobora la importancia del título como elemento contextualizador. En un estudio realizado con anglo-parlantes, Bransford y Johnson (1972) midieron cómo afectaba a la comprensión de un texto el informar al sujeto del contexto previamente para concluir que aquellos sujetos que conocían el título antes de escuchar el texto, llegaban a una comprensión mucho mayor de éste. En sus propias palabras, «[the title's] critical role appears to be in helping [subjects] create contexts that can be used to comprehend the passages in the first place» (p. 724). A la misma conclusión llegaban Schwarz y Flammer (1981) en su investigación con el cuento tradicional. En este caso, los sujetos del experimento leían un cuento con o sin un título temático, que es aquel que contiene al menos una o dos palabras que apuntan directamente a los contenidos del texto que sigue.

\section{DESCRIPCIÓN DE LA INVESTIGACIÓN}

\subsection{Características del texto}

Como decíamos en la sección anterior, este estudio se centra en el análisis del título como componente básico para la activación de los esquemas necesarios -y por tanto para la comprensión satisfactoria del texto- en la lectura en segundas lenguas. En concreto, nos 
centramos en la lectura de un texto periodístico auténtico en lengua inglesa por parte de hablantes de inglés como segunda lengua cuya primera lengua es el español, con el fin de comprobar hasta qué punto es beneficiosa la presencia del título y qué efectos causa su ausencia.

Para ello, se eligió una noticia principalmente por una razón: en el discurso periodístico, el título es parte inherente de la estructura del texto, en el cual tiene un peso específico y una función definida, que es normalmente la de anticipar el tema central de la noticia ${ }^{4}$. Generalmente, las noticias en inglés siguen un esquema prototípico o superestructura (Van Dijk, 1990), bajo la cual se engloban los distintos apartados, que son (en la traducción de González Rodríguez, 2001): (a) el sumario, que se subdivide en el encabezamiento y el primer párrafo del cuerpo principal; (b), los acontecimientos: por un lado el acontecimiento principal y por otro los antecedentes de la noticia; (c) las consecuencias y (d) los comentarios. En el esquema de Van Dijk, el título al que nos referimos en esta investigación corresponde al encabezamiento de la sección (a). Creemos que prescindir del título en una noticia, más que en otros tipos de discurso, supone trastocar la estructura del texto y omitir la presentación de la información contenida en él, impidiendo, antes de la lectura, la activación de los esquemas necesarios para comprenderlo ${ }^{5}$.

A pesar de que se trata de un texto periodístico auténtico, lo cual puede hacer suponer que su léxico es complejo, un estudio piloto previo con 10 sujetos del mismo nivel de inglés que los sujetos de esta investigación reveló que los primeros desconocían sólo el 4’3\% del total de palabras. Por tanto, la densidad de palabras desconocidas prevista se encuentra entre los siguientes parámetros: se supera el $2 \%$ que parece garantía de comprensión (Nation y Coady, 1988) con el fin de permitir una posible diferencia significativa entre la lectura con o sin contextualización previa; sin embargo, no se alcanza el 6\% que parece ser el límite aconsejable de palabras desconocidas en textos auténticos (Nation, 1990: 116).

El formato en que se presentó la noticia trataba de asemejarse al periodístico con el fin de que el alumno pudiera identificarlo como tal y enfrentarse a él de acuerdo con las expectativas que tiene acerca de un texto de este género. Una copia del texto y de la actividad de comprensión lectora tal y como les fue entregado a los sujetos de la investigación se puede encontrar en el apéndice de este artículo.

\subsection{Los sujetos de la investigación}

La investigación se llevó a cabo con un total de 90 alumnos pertenecientes a dos grupos de primer curso de la especialidad de Educación Primaria de la diplomatura de Magisterio, todos ellos hablantes nativos de español que cursaban una asignatura troncal de especialidad de lengua inglesa de nivel intermedio. A estos 90 alumnos se los dividió de manera aleatoria en tres grupos diferentes de 30 alumnos, con el fin de asignar a cada uno de ellos el texto acompañado o no de contextualización previa en inglés o en español.

${ }^{4}$ El titular de la noticia también tiene a veces la función de llamar la atención del lector y otras veces su objetivo es presentar el tema a tratar de manera más general que informativa; así, los titulares se pueden clasificar en los que sí tienen la función de anticipar la noticia a modo de resumen, llamados «informativos» y los que persiguen estos dos últimos objetivos: «llamativos» y «genéricos» (León, 1996).

${ }^{5}$ El texto seleccionado pertenece a la publicación local «Chronicle», de Montana, Estados Unidos. 


\subsection{Procedimiento seguido para la recogida de datos}

A los sujetos, que, como decíamos anteriormente, estaban divididos en tres grupos, se les dio el texto y la actividad de comprensión lectora de acuerdo con el siguiente criterio: los grupos A y B recibieron el texto con el título, en español y en inglés respectivamente; el grupo C recibió el texto sin título.

Previamente a la entrega del texto, para tener la certeza de que la atención de los sujetos elegidos para ver el título se centraba durante unos instantes exclusivamente en éste y se activaban los esquemas necesarios, se proporcionó a los grupos A y B la siguiente información:

- Grupo A: «Traducido al español, el título del texto que vas a leer a continuación es: "Adolescente cambia su nombre por Pesca de la Trucha en América."»

- Grupo B: «El título del texto que vas a leer a continuación es: "Teen changes his name to Trout Fishing in America."»

Por otro lado, al grupo C se le entregó el texto en inglés, sin ninguna explicación ni aclaración.

Los alumnos disponían de 20 minutos para leer el texto y responder a las preguntas de comprensión lectora que lo acompañaban, todas ellas del tipo multiple choice con cuatro posibles respuestas (A, B, C y D), de las cuales sólo una era correcta.

Un primer tratamiento de los datos reveló que una de las preguntas de la actividad de comprensión lectora (concretamente la número dos) había sido diseñada incorrectamente puesto que permitía dos respuestas acertadas y tuvo que ser invalidada; Por tanto, la puntuación máxima de la actividad fue 9 y no 10 .

\section{Resultados}

Los noventa tests obtenidos (treinta por cada grupo) fueron analizados estadísticamente para averiguar: (a) si existía relación significativa entre la contextualización mediante la exposición al título y un mejor rendimiento en la actividad de comprensión lectora -entre los resultados de los grupos A y B por un lado, y los del grupo C por otro- y (b), si era posible encontrar también una diferencia significativa entre los datos de los grupos A y B, es decir, bien al enfrentarse al texto previa exposición al título en la lengua materna o bien, al hacerlo en la segunda lengua.

En cuanto al apartado (a), como se puede observar en la tabla 1, la media de respuestas correctas obtenida por los grupos A (título en español) y B (título en inglés) se puede considerar significativamente mayor que la media de C (sin título); concretamente en el caso de la diferencia entre los grupos A y $\mathrm{C}$, el primero aventaja al segundo en prácticamente un punto y medio (6,23 sobre 9 frente a 4,87 sobre 9 respectivamente). En la tabla 1 también se ve reflejado que las medias extraídas para cada grupo son representativas de los resultados individuales de los sujetos, como muestra la desviación típica obtenida en los tres casos, especialmente en los grupos $\mathrm{A}$ y $\mathrm{C}$, con resultados ciertamente similares $(\mathrm{dtA}= \pm 1,3$ y dtC $= \pm 1,32)$. 
Tabla 1: Respuestas acertadas en la actividad de comprensión lectora

\begin{tabular}{lcc} 
& Media aritmética & Desviación típica \\
\hline Grupo A & 6,23 & $\pm 1,3$ \\
Grupo B & 6,03 & $\pm 1,4$ \\
Grupo C & 4,87 & $\pm 1,32$ \\
\hline
\end{tabular}

La diferencia entre, por una parte, los grupos A y B y por otra, el grupo C se puede apreciar más claramente en la gráfica 1:

Gráfica 1. Respuestas acertadas en la actividad de comprensión lectora

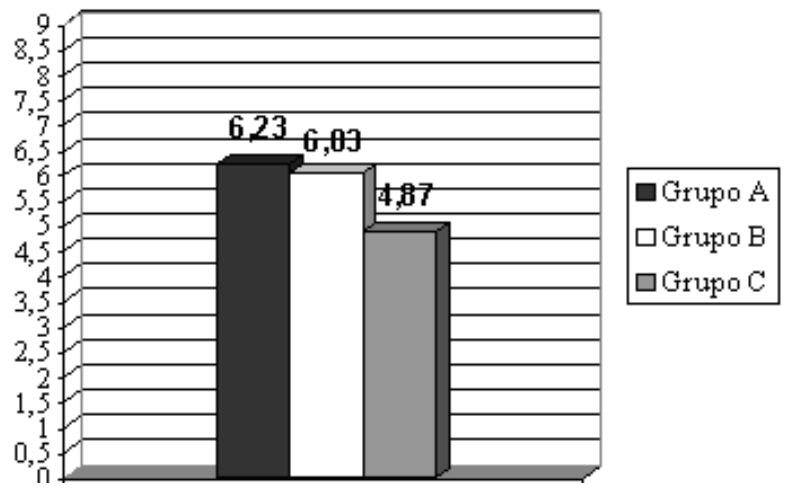

Por otro lado, tratando de dar respuesta a la pregunta planteada en el apartado (b) al principio de esta sección, es decir, centrándonos en los grupos A y B y en la comparación de sus medias de contestaciones correctas, vemos que, efectivamente, los datos arrojan una diferencia entre la actuación de ambos grupos, siendo en general aquellos sujetos que leen el título en español los que obtienen un mejor resultado (6,23 de media en el grupo A frente a 6,03 del grupo B), pero consideramos que la diferencia no es significativa como para afirmar de forma concluyente que la lectura del título en la lengua materna facilita la activación de los esquemas necesarios más eficazmente que su lectura en la segunda lengua.

\section{Conclusiones}

En primer lugar, esta línea de investigación tiene varias implicaciones metodológicas para la enseñanza de segundas lenguas en general, y de $T E F L^{6}$ en particular. Por una parte,

${ }^{6}$ Siglas en inglés de Teaching English as a Foreign Language; en español: 'la enseñanza del inglés como lengua extranjera'. 
desde un punto de vista general, la aplicación en el aula de la Teoría del Esquema que se ha presentado en estas líneas conlleva una percepción muy particular del proceso de lectura y de las posibles complicaciones derivadas de éste. Según este enfoque, antes de la lectura resulta útil considerar qué esquemas es necesario activar para la comprensión del texto y distinguir entre esquemas lingüísticos o formales, relacionados directamente con el nivel de competencia lingüística del alumno, y esquemas de contenido, que pueden ser propios de la cultura de la segunda lengua y desconocidos para el lector no nativo; la metodología que se seguirá en el aula se verá condicionada por el resultado de este análisis. Además, al alumno se le asigna un papel fundamental en el proceso, al reconocer la relevancia de sus conocimientos previos en la interpretación del texto. Al no estar prefijados de antemano ni el acierto ni el fracaso de las respuestas del alumno, la situación de aprendizaje será más flexible.

Por otra parte, en estas líneas hemos probado que la presencia del título parece guiar efectivamente al lector de una segunda lengua en su búsqueda de esquemas para su activación y la subsiguiente comprensión satisfactoria del texto. No parece arriesgado afirmar, entonces, que la preparación a la lectura ayuda al aprendiz de segundas lenguas en la comprensión y, por tanto, conduce al aprendizaje. El análisis del grado de incidencia en la comprensión lectora del título como recurso metodológico y de otros recursos de pre-lectura podría facilitar el diseño de una metodología que contemple la activación de los esquemas necesarios de manera controlada para el desarrollo de estrategias de lectura y de la destreza de lectura en general. Un estudio cuantitativo longitudinal en el que el grupo experimental -expuesto a un trabajo sistemático con actividades de pre-lectura- y el de control fueran evaluados en base a su comprensión lectora aportaría más datos en esta dirección. También sería interesante medir las consecuencias que tiene para el alumno su preparación consciente para la activación de los esquemas adecuados.

Finalmente, una continuación de la investigación con los sesenta participantes de los grupos A y B podría arrojar más datos sobre la cuestión, sin resolver en este artículo, de si la contextualización previa en la lengua materna conduce sistemáticamente a una mayor comprensión que si tiene lugar en la segunda lengua. La lectura de un texto de características similares a las del texto del presente estudio por parte de los mismos sujetos -esta vez los del grupo A leyendo el título en inglés y los del grupo B, con el título traducido al español-, y un posterior análisis de correlación entre ambas variables (lectura del título en español y en inglés) cuando se dan en un mismo sujeto, ayudaría a delimitar el grado de relación entre las dos posibilidades y contribuiría al debate sobre la pertinencia del uso de la lengua materna en las clases de segundas lenguas.

\section{REFERENCIAS Bibliográficas}

Alderson, J. C. y Urquhart, A. H. (1983). "The effect of background discipline on comprehension: a pilot study", en A. Hughes y D. Porter (eds.), Current Developments in Language Testing. Londres: Academic, 121-127.

Anderson, R. C. y Pearson, P. D. (1984). "A schema-theoretic view of basic processes in reading comprehension”, en P. D. Pearson (ed.), A Handbook of Reading Research. Londres: Longman, 37-55. 
Block, E. (1986). "The comprehension strategies of second language readers", en TESOL Quarterly, 20, 3: 463-494.

Bransford, J. D. y Johnson, M. K. (1972). "Contextual prerequisites for understanding: some investigations of comprehension and recall", en Journal of Verbal Learning and Verbal Behavior, 11: 717-726.

Carrell, P. L. (1983). "Some issues in studying the role of schemata, or background knowledge, in second language comprehension". Ponencia presentada en la XVII Convención anual TESOL, Toronto, Canadá, Marzo 1983.

Carrell, P. L. y Eisterhold, J. C. (1983). "Schema theory and ESL reading pedagogy", en TESOL Quarterly, 17: 553-573.

Carroll, J. B. (1978). "The nature of the reading process", en L. J. Chapman y P. Czerniewska (eds.), Reading. From Process to Practice. Londres: Routledge \& Kegan Paul y Open University Press, 95-105.

Devitt, S. (1997). "Interacting with authentic texts: multilayered processes", en Modern Language Journal, 81, 4: 457-469.

Dijk, T. van (1990). La Noticia como Discurso. Barcelona: Paidós.

Eskey, D. (1988). "Holding in the bottom: an interactive approach to the language problems of second language readers", en P. L. Carrell, J. Devine y D. E. Eskey (eds.), Interactive Approaches to Second Language Reading. Cambridge: Cambridge University Press, 93100.

Fecteau, M. L. (1999). "First- and second-language reading comprehension of literary texts", en Modern Language Journal, 83, 4: 475-493.

Fillmore, C. (1981). "Ideal readers and real readers", en D. Tannen (ed.), Analyzing Discourse: Text and Talk. Georgetown University Round Table on Language and Linguistics. Washington D. C.: Georgetown University Press, 248-270.

González Rodríguez, M. J. (2001). "La estructura del relato periodístico informativo en los diarios nacionales británicos”, en Revista Canaria de Estudios Ingleses, 42: 289-307.

Goodman, K. y Goodman, Y. (1978). "Learning about psycholinguistic processes by analyzing oral reading”, en L. J. Chapman y P. Czierniewska (eds.), Reading. From Process to Practice. Londres: Routledge \& Kegan Paul y Open University Press, 126-145.

León, J. A. (1996). Prensa y Educación: un Enfoque Cognitivo. Buenos Aires: Aique.

Nation, I. S. P. (1990). Teaching and Learning Vocabulary. Nueva York: Newbury House.

Nation, I. S. P. y Coady, J. (1988). "Vocabulary and reading”", en R. Carter y M. McCarthy (eds.), Vocabulary and Language Teaching. Londres: Longman, 97-109.

Schwarz, M. N. K. y Flammer, A. (1981). "Text structure and title: effects on comprehension and recall", en Journal of Verbal Learning and Verbal Behavior, 20: 61-66.

Silberstein, S. (1994). Techniques and Resources in Teaching Reading. Nueva York: Oxford University Press. 


\section{APÉNDICE}

\section{a. El texto:}

\section{TEEN CHANGES HIS NAME TO TROUT FISHING IN AMERICA}

CARPINTERIA, Calif.- A teen-ager casting about for a new name has reeled in a golden one: Trout Fishing in America.

The 17-year-old wanted to do something different for his upcoming graduation from Carpinteria High School. So he went to court last week to legally change his name from Peter Eastman, Jr. to Trout Fishing in America.

Trout said he decided to change his name to break away from tradition. "I am just saying I am not a kid anymore. I want to be my own person".

The name was inspired by Richard Brautigan's 1967 counter-culture classic, "Trout Fishing in America".

\section{b. Actividad de comprensión lectora:} Reading comprehension activity.

Contesta a las siguientes preguntas de acuerdo con el texto que acabas de leer.

1. «Trout» es:
a. un nombre.
b. un pescador.
c. un pez.
d. un libro

2. ¿Qué entiendes por «reeled in» en la línea 3?
a. olvidar.
b. oír.
c. encontrar.
d. pescar.

3. ¿Qué es «upcoming» en la línea 7?
a. un tipo de graduación.
b. poco frecuente.
c. próxima.
d. algo relacionado con la carpintería.
4. El chico cambió su nombre porque:
a. le gustaba mucho pescar
b. no le gustaba su nombre anterior.
c. quería impresionar a la gente en su graduación.
d. quería convertirse en alguien especial.

5. Richard Brautigan es:
a. un músico clásico.
b. el autor de un famoso libro.
c. un pescador famoso.
d. el nombre que quería ponerse el protagonista.

6. ¿Quién es «He» en la línea 25?
a. el adolescente.
b. Peter Eastman.

"I really like the book", Trout said. "He looks at the world in a way I like".

Peter Eastman, Sr. said he supports his son's decision, but said that the family torch has been passed to a fish.

Still, the senior Eastman paid the $\$ 182$ name-change filling fee as a graduation present for his son. It should become official in a couple of weeks and the name Trout Fishing in America should appear on his diploma in June.

The boy's mother isn't sure what to call her son. "Trout Fishing in America is a little long" Addie Green said. c. Richard Brautigan.

d. Trout.

7. ¿Qué información tenemos acerca del padre? a. está descontento con la decisión de su hijo. b. está preocupado por la legalidad del nombre.

c. apoya a su hijo y además le paga el cambio de nombre.

d. está loco puesto que cree que su hijo es un pez.

8. La expresión «To pass the family torch» en las líneas 29-30 quiere decir:

a. hacer algo diferente e inesperado.

b. pasar las tradiciones familiares a las nuevas generaciones.

c. pasar el nombre de familia a la nueva generación.

d. convertirse de chico en pez.

9. El nuevo nombre:
a. ya es oficial.
b. es sólo un mote.
c. no es oficial pero lo será pronto.
d. no puede hacerse oficial.

10. La madre:
a. está preocupada.
b. está desconcertada.
c. no cree que el diploma deba tener un nombre tan largo.
d. cree que el diploma no será válido por tener un nombre tan largo. 